Нечитайло И.А.

Санкт-Петербургский политехнический университет Петра Великого, Санкт-Петербург, Российская Федерация; nechitajlo_ia@spbstu.ru

\title{
РЕФЕРАТ
}

Цель. Цель исследования состояла в оценке применимости в стратегическом анализе подхода, известного как пять направляющих шагов теории ограничений систем.

Методы. Использованы методы детерминированного факторного анализа и финансовой математики, в частности, методы оценки реальных опционов.

Результаты. Показано, что в некоторых ситуациях увеличение рыночной добавленной стоимости бизнеса может достигаться только рядом инвестиционных проектов, приводящих к последовательному снятию ограничений и созданию положительных синергетических эффектов, а также к появлению реальных опционов. В таких ситуациях оценка отдельных проектов без учета последующих планируемых и возможных стратегических преобразований может вводить в заблуждение относительно их ценности.

Выводы. Основным объектом оценки в стратегическом анализе должна быть стратегия как ряд будущих преобразований с учетом порождаемых ими реальных опционов. Поэтому оценка проектов отдельных стратегических преобразований в последовательности, предписываемой теорией ограничений систем, может приводить к занижению их ценности и необоснованному отклонению. Вместе с тем, использование теории ограничений систем страхует от инвестирования в проекты, не приводящие к ослаблению или снятию в стратегической перспективе ограничений бизнеса.

Ключевые слова: теория ограничений систем, ограничение системы, стратегия, инвестиционный проект, реальный опцион

Для цитирования: Нечитайло И. А. Пять направляющих шагов теории ограничений систем: проблемы применения в стратегическом анализе // Управленческое консультирование. 2020. № 6. С. 97-109.

\section{The Five Focusing Steps of the Theory of Constraints: Problems of Application in Strategic Analysis}

\section{Igor A. Nechitaylo}

Peter the Great St. Petersburg Polytechnic University, Saint-Petersburg, Russian Federation; nechitajlo_ia@spbstu.ru

\section{ABSTRACT}

Objectives. We identify and investigate circumstances that limit an application of the Five Focusing Steps of the Theory of Constraints in the strategic analysis of investment projects effectiveness.

Methods. The methods of factor analysis and financial mathematics including methods for evaluation real options are used.

Results. It is shown that in some cases, an increase in business value can be created only by a combination of a number of investment projects, leading to consistent removal of constraints and arising of useful synergistic effects. It is also shown that a value of a project can be determined by changes in the strategy that the company will have the opportunity to make if project is implemented. In such cases, their evaluation without taking into account subsequent decisions may distort their true value.

Conclusions and Relevance. We concluded that the main object of evaluation in strategic analysis should be a strategy as a series of future transformations, taking into account the 
real options generated by them. Therefore, the evaluation of single projects on the base of the Theory of Constraints can lead the rejection of valuable projects due to distortion of their true value. At the same time an application of the Five Focusing Steps in strategic analysis protect against investment in projects with negative net present value.

Keywords: theory of constraints, system constraints, strategy, invest project, real option

For citing: Nechitaylo I. A. The Five Focusing Steps of the Theory of Constraints: Problems of Application in Strategic Analysis // Administrative consulting. 2020. No. 6. P. 97-109.

\section{Введение}

Одним из наиболее спорных аспектов стратегического анализа является процесс формирования стратегии. Об этом можно судить хотя бы по отсутствию единой точки зрения на то, является ли появление стратегии в большей мере целенаправленным процессом планирования или стихийным процессом, что отмечается Р. Грантом ${ }^{1}$. Если понимать под стратегией последовательность действий, приводящих к качественному изменению параметров управляемой системы, то наличие данной дилеммы ставит вопрос о подходе к выбору их последовательности.

Несмотря на разработанность проблематики анализа конкурентной среды и потенциала корпораций в стратегическом менеджменте, строгое обоснование этого подхода оказалось затруднительным. В связи с этим заслуживает внимания подход к управлению хозяйственными системами, сформулированный в теории ограничений систем (ТОC). Согласно данной теории оптимизация большинства процессов в системе (т.е. локальная оптимизация) чаще всего не приводит к оптимизации системы в целом (что неявно предполагается в традиционном подходе к менеджменту). Напротив, в каждый момент улучшение результата деятельности хозяйственной системы как совокупности связанных процессов возможно только вследствие улучшения условий лишь немногих из них. Эти условия деятельности называются ограничениями системы. Таким образом, в ТОС управление системой сводится к управлению ее ограничениями.

Изначально данная теория была разработана для решения задач оперативного управления производством Э. Голдраттом [13-16]. В дальнейшем разработанные им принципы и метод управления цепями поставок «барабан-буфер-канат» были развиты другими авторами. В частности, исследовались влияние вариабельности показателей операционных процессов на показатель прохода системы в сложных случаях «игры в кости» [18] и возможности снижения отрицательного действия эффекта хлыста в цепях поставок при помощи ТОС [25]. Предпринимались попытки модификации метода «барабан-буфер-канат», наиболее значимой из которых можно считать исследование У. Детмера и Э. Шрагенхайма [3]. В последнее время большое внимание уделялось возможностям синтеза ТОС с концепциями бережливого производства и «шести сигм», исследованных Б. Спроуллом [26], Р. Диасом, Л. Сильвой, А. Тенерой [11], М.В. Хайруллиной, О.А. Кислицыной, А. В. Чуваевым [7] и Е.С. Балашовой [1]. Кроме того, обобщался опыт внедрения ТОС в отдельных областях деятельности, начиная с предприятий мебельной и фармацевтической промышленности [17; 21], птицеферм [10] и заканчивая туристическим бизнесом [22].

Помимо управления производством и цепями поставок ТОС нашла применение в проектном менеджменте. На ее основе Э. Голдраттом был разработан метод критической цепи, развитый Л. Личем [6] и нашедший широкое применение в ряде областей, в частности, в управлении строительными проектами [23]. Также

\footnotetext{
${ }^{1}$ Грант Р. М. Современный стратегический анализ. СПб. : Питер, 2018.
} 
известен вклад ТОС в преодоление ограничений классического управленческого учета, исследованный С. Бреггом [9] и Т. Корбеттом [5].

Наиболее же полная систематизация положений ТОС, значимых для построения стратегии организации, была предпринята У. Детмером [2], а их универсальность показана Э. Шрагенхаймом [8], исследовавшим их применимость в самых различных областях деятельности. Их квинтэссенцией можно считать подход, позволяющий упорядочивать работу с ограничениями хозяйственной системы и получивший название пяти направляющих шагов ТОС. Несмотря на сложность его применения к некоторым типам ограничений (а именно рыночным и нефизическим ограничениям), исследованных П. Преториусом [24], М. Гуптой и др. [19], этот подход наиболее полно выражает логику ТОС. Согласно ему в первую очередь необходимо установить основное ограничение системы. Далее обеспечить максимально эффективное использование имеющегося потенциала этого ограничения и подчинить ему работу остальных элементов системы. И только когда эти шаги выполнены, можно приступать к оценке целесообразности расширения ограничения системы, в том числе за счет капиталоемких мероприятий. Если в результате одного из этих действий существующее ограничение будет устранено, следует найти новое ограничение системы и повторить этот цикл снова.

Можно согласиться с У. Детмером и Э. Шрагенхаймом [3, с. 65], что использование существующих ограничений системы и подчинение им работы остальных элементов можно отнести к тактическим шагам, в то время как нахождение и расширение ограничений - к шагам стратегическим. Поэтому с позиций ТОС бизнесстратегию организации можно представить как ряд капиталоемких мероприятий, направленных на последовательное снятие ограничений системы и повышение эффективности их использования.

Исходя из этого оценка бизнес-стратегии организации, на первый взгляд, может быть представлена как сумма чистых приведенных стоимостей (NPV) инвестиционных проектов, анализ которых проводится на цепной основе в предписанной ТОС последовательности. Проблема в том, что несмотря на логичность такого подхода, расчет NPV проектов на цепной основе в некоторых ситуациях может вводить в заблуждение относительно их действительной ценности. А это может приводить к необоснованному отклонению стратегии в целом и упущенным выгодам для организации. Для предотвращения таких упущенных выгод при применении ТОС в стратегическом анализе целесообразно выделить основные типы ситуаций, в которых могла бы проявиться ограниченность подобного подхода. Для этого необходимо исследовать влияние ограничивающих факторов бизнеса на изменение его стоимости в ситуациях со связанными инвестиционными проектами и реальными опционами.

\section{Материалы и методы исследования}

Для решения поставленной задачи рассмотрим несколько гипотетических хозяйственных ситуаций, которые могли бы возникнуть при реализации двух связанных инвестиционных проектов на промышленном предприятии с однопродуктовым производством. В этом производстве однородное сырье проходит переработку на участке $X$, а полученный из него промежуточный продукт перерабатывается на участке $Y$ в конечный продукт. Рынок промежуточного продукта отсутствует, а рынок конечного продукта представляет собой конкурентный рынок с настолько большим количеством продавцов и покупателей, что данное промышленное предприятие можно рассматривать как ценополучателя. На рынке сырья данное предприятие также не способно оказывать существенного влияния на цену изменением объема его закупок. С позиций директ-костинга все затраты данного предприятия, за ис- 
ключением затрат сырья, можно рассматривать как условно-постоянные расходы, изменяющиеся в большей мере в результате изменения производственных мощностей, чем под влиянием изменения их загрузки.

На предприятии существует по одному проекту модернизации производства на каждом участке. С технической точки зрения модернизация производства на участке $X$ может выполняться независимо от проведения модернизации на участке $Y$ и наоборот. Таким образом, у руководства предприятия существует 4 варианта действий: не предпринимать изменений и поддерживать сложившиеся параметры производства (базисная стратегия); провести модернизацию на участке $X$ (проект $X$ ); провести модернизацию на участке $Y$ (проект $Y$ ); провести модернизацию на обоих участках (комплекс проектов $X$ и $Y$ ). Причем последний вариант предполагает одновременную реализацию этих двух проектов.

В целях упрощения предположим, что время проведения модернизации производства на участках $X$ и $Y$ пренебрежимо мало и проекты начнут давать результат в первый месяц после их принятия. При этом срок использования основного технологического оборудования на участках $X$ и $Y$ до следующих крупных вложений одинаков и составит 8 лет, которые примем за горизонт планирования, что обеспечит сопоставимость проектов. В общем же случае можно было бы использовать метод бесконечного цепного повтора [4], который позволяет, во-первых, сопоставлять проекты с разными сроками жизни, во-вторых, автоматически включать в оценку $N P V$ терминальную стоимость проектов, которую мы не будем учитывать в расчетах.

Также в целях упрощения предполагается, что при любом варианте действий нормы затрат сырья и выхода конечного продукта из полуфабриката, цены на сырье и конечный продукт (табл. 1), а также производственная мощность участков (табл. 2) будут оставаться неизменными на горизонте планирования. Спрос на продукцию организации в первый год ожидается на уровне 700 тыс. т конечного продукта, во второй год - 720 тыс. т и начиная с третьего года -730 тыс. т в год. Экономия на условно-постоянных расходах (без учета амортизации оборудования) вследствие модернизации производства составит: по проекту $X-30$ млн руб. в год, по проекту $Y-20$ млн руб. в год.

Исходя из этих предпосылок чистая операционная прибыль после налогообложения в системе «директ-костинг» может быть представлена в виде следующей модели:

$$
O P_{t}=(q(t) \times(p-r v)-F C-D) \times(1-s),
$$

где $O P_{t}$ - операционная прибыль после налогообложения в году $t$, млн руб.; $q(t)$ - объем продаж в году $t$ на горизонте планирования, тыс. т; $p$ - цена тонны конечной продукции, тыс. руб.; $r$ - норма расхода полуфабриката на тонну конечного продукта на участке $Y$, т; $v$ - удельные затраты сырья на тонну полуфабриката на участке $X$, тыс. руб.; $F C$ - условно-постоянные расходы предприятия (кроме амортизации), млн руб.; $D$ - амортизация основных средств в управленческом и налоговом учете, млн руб.; $s$ - ставка налога на прибыль

Из табл. 1 и прогноза спроса видно, что основным ограничивающим фактором системы на горизонте планирования при базисной стратегии станет производственная мощность участка $Y$. Следовательно в соответствии с ТОС анализ целесообразности изменений в системе следует начинать с оценки $N P V$ проекта мо-

1 Далее базисные значения этих показателей будем обозначать нижним индексом 0, а значения по проектам $X$ и $Y$ и их комплексу - нижними индексами $X, Y$ и $Y X$ соответственно. Влияние на прибыль факторов $v, r, q$ также будем обозначать соответствующим нижним индексом. 
Цены и нормы затрат при разных вариантах действий руководства предприятия

Table 1. Prices and cost rates with different corporate strategies

\begin{tabular}{|c|c|c|c|c|}
\hline Наименование показателя & 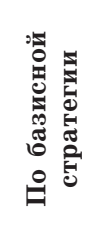 &  & 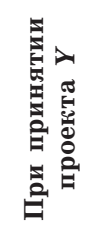 & 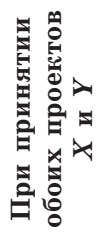 \\
\hline Цена т конечной продукции, тыс. руб. & 10,00 & 10,00 & 10,00 & 10,00 \\
\hline $\begin{array}{l}\text { Норма расхода сырья на т полуфабриката на } \\
\text { участке } X \text {, тыс. руб. }\end{array}$ & 3,00 & 2,90 & 3,00 & 2,90 \\
\hline $\begin{array}{l}\text { Норма выхода конечного продукта из } \\
\text { полуфабриката на участке Y (\% к весу } \\
\text { полуфабриката) }\end{array}$ & 80,00 & 81,00 & 82,00 & 82,50 \\
\hline $\begin{array}{l}\text { Норма расхода полуфабриката на производ- } \\
\text { ство т конечного продукта на участке } Y, \text { т }\end{array}$ & 1,250 & 1,235 & 1,220 & 1,212 \\
\hline $\begin{array}{l}\text { Удельная маржинальная прибыль с т конеч- } \\
\text { ной продукции, тыс. руб. }\end{array}$ & 6,250 & 6,418 & 6,340 & 6,485 \\
\hline
\end{tabular}

Источник: авторская разработка.

Таблица 2

\section{Производственные мощности при разных вариантах действий руководства предприятия}

Table 2. Production capacities with different corporate strategies

\begin{tabular}{|l|c|c|c|c|}
\hline \multicolumn{1}{|c|}{ Наименование показателя } & . & & \\
& & & \\
\hline $\begin{array}{l}\text { Производственная мощность участка } X \text { в весовом } \\
\text { эквиваленте полуфабриката, тыс. т }\end{array}$ & 850 & 1000 & 850 & 1000 \\
\hline $\begin{array}{l}\text { Производственная мощность участка Y в весовом } \\
\text { эквиваленте полуфабриката, тыс. т }\end{array}$ & 800 & 800 & 950 & 950 \\
\hline $\begin{array}{l}\text { Производственная мощность предприятия в весо- } \\
\text { вом эквиваленте полуфабриката, тыс. т }\end{array}$ & 800 & 800 & 850 & 950 \\
\hline $\begin{array}{l}\text { Производственная мощность предприятия в весо- } \\
\text { вом эквиваленте конечного продукта, тыс. т }\end{array}$ & 640 & 648 & 697 & 783,8 \\
\hline
\end{tabular}

Источник: авторская разработка.

дернизации на этом участке. Для этого необходимо оценить изменение будущих денежных потоков предприятия на горизонте планирования вследствие принятия этого проекта. Наиболее существенной и сложной составляющей этой величины будет изменение маржинальной прибыли после налогообложения, которое будет определяться двумя факторами. Во-первых, это снижение нормы затрат полуфабриката на единицу конечной продукции за счет увеличения нормы выхода с 80\% 
до $82 \%$ в связи с изменением технологии производства. Во-вторых, это увеличение производственной мощности за счет увеличения нормы выхода конечного продукта и расширения емкости технологического оборудования, что позволит удовлетворять дополнительный спрос в перспективе и увеличить объем продаж на 57 тыс. т начиная с первого года. Влияние этих факторов на прибыль может быть оценено следующим образом:

$$
\begin{gathered}
\Delta O P_{Y r t}=-q_{0}(t)\left(r Y-r_{0}\right) v_{0}(1-s), \\
\Delta O P_{Y q t}=\left(q_{Y(t)}-q_{0}(t)\right)\left(p-r_{Y v 0}\right)(1-s) .
\end{gathered}
$$

Сложив эти величины с экономией на условно-постоянных расходах и на налоге на прибыль за счет увеличения амортизации основных средств, получим денежный поток по проекту $Y$ :

$$
C F_{Y t}=\Delta O P_{Y r t}+\Delta O P_{Y q t}-\left(F C_{\mathrm{Y}}-F C_{0}\right)(1-s)+\left(\Delta Y-D_{0}\right) \mathrm{s},
$$

где $C F_{Y t}$ - денежный поток по проекту $Y$ за год $t$.

В случае неотрицательной NPV проекта $Y$ он должен быть включен в стратегический план. После этого основным системным ограничением на горизонте планирования становится производственная мощность оборудования на участке $X$. Поэтому далее в соответствии с логикой TOC необходимо оценить NPV проекта $X$. Поскольку проект $X$ оценивается на цепной основе, прирост маржинальной прибыли по проекту $X$ должен включать все синергетические эффекты комплекса проектов, в том числе и от снятия нового ограничения по мощности на этом участке. При этом прирост данного показателя будет определяться тремя факторами. Во-первых, это снижение удельных затрат сырья на участке $X$ с 3,00 до 2,9 тыс. руб. на т полуфабриката. Этот эффект частично мог бы быть получен при автономной реализации проекта $X$, но при поэтапном построении стратегии должен быть оценен в расчете на достигнутый на первом этапе объем продаж. Во-вторых, это дополнительное снижение нормы затрат полуфабриката на единицу конечной продукции на участке $Y$ за счет увеличения нормы выхода с $82 \%$ до $82,5 \%$ в связи с улучшением качества полуфабриката на участке $X$. В-третьих, это дополнительное увеличение производственной мощности за счет дальнейшего увеличения нормы выхода конечного продукта и расширения емкости технологического оборудования. Это позволит удовлетворять больший спрос и увеличить объем продаж на 3 тыс. т в первый год, 23 тыс. т во второй год и на 33 тыс. т начиная с третьего года. Оценка влияния этих факторов на прибыль может быть получена следующим образом:

$$
\begin{gathered}
\Delta O P_{X v}=-q_{Y}(t) r_{Y}\left(v_{X}-v_{0}\right)(1-s), \\
\Delta O P_{X r t}=-q_{Y}(t)\left(r_{Y X}-r_{Y}\right) v_{X}(1-s), \\
\Delta O P_{X q t}=\left(q_{Y X}(t)-q_{Y}(t)\right)\left(p-R_{Y X} v_{X}\right)(1-s) .
\end{gathered}
$$

Если бы проект $X$ рассматривался изолированно от проекта $Y$, прирост маржинальной прибыли под влиянием этих факторов определялся бы так:

$$
\begin{gathered}
\Delta O P_{X v}^{*}=-q_{Y}(t) r_{Y}\left(v_{X}-v_{0}\right)(1-s), \\
\Delta O P_{X r t}^{*}=-q_{Y}(t)\left(r_{Y X}-r_{Y}\right) v_{X}(1-s), \\
\Delta O P_{X q t}^{*}=\left(q_{Y X}(t)-q_{Y}(t)\right)\left(p-R_{Y X} v_{X}\right)(1-s) .
\end{gathered}
$$


Исходя из этого синергетический эффект от проекта $X$ в контексте принятого проекта $Y$ может быть определен попарным сопоставлением соответствующих величин, определяемых по формулам (2-4) и (5-7). В остальном денежные потоки по проекту $X$ будут складываться из схожих составляющих, что и по проекту $Y$ :

$$
C F_{X t}=\Delta O P_{X v t}+\Delta O P_{X r t}+\Delta O P_{X q t}-\left(F C_{X}-F C_{0}\right)(1-s)+\left(D_{X}-D_{0}\right) s,
$$

где $C F_{X t}-$ денежный поток по проекту $X$ за год $t$.

Таким образом, NPV комплекса из двух инвестиционных проектов с позиций TOC может быть представлена как сумма NPV от реализации стратегии из двух организационно-технических мероприятий, в которой синергетический эффект от совместного их выполнения включается в NPV второго мероприятия. При определении NPV этих проектов будем исходить из предположения, что величины будущих денежных потоков имеют симметричное распределение, поэтому за математическое ожидание денежного потока может быть взято его медианное значение, которое и потребуется определить по вышеприведенному алгоритму. При этом дисконтирование проводится по ставке $10 \%$ с непрерывным начислением.

В целях выявления ограничений применимости ТОС в стратегическом анализе проведем оценку NPV каждого из двух проектов в соответствии с вышеизложенным алгоритмом в трех ситуациях. Они идентичны во всем за исключением, во-первых, величины стартовых вложений в модернизацию производства на участках $X$ и $Y$ и, соответственно, амортизации оборудования, во-вторых, реальных опционов. Стартовые вложения в проекты $X$ и $Y$ составят в ситуации 1: 1350 и 1950 млн руб.; в ситуации 2: 1050 и 2250 млн руб.; в ситуации 3: 1550 и 1950 млн руб. соответственно. Принципиальное отличие ситуации 3 заключается в том, что помимо ранее рассмотренных эффектов модернизация производства на участке $X$ даст возможность замены традиционного сырья его недавно появившимся на рынке аналогом. Ожидаемое среднее значение цены на это сырье на горизонте планирования составляет 2,8 тыс. руб. за т. Тем не менее, цены на него неустойчивы, а их стабилизация ожидается через два года. В случае стабилизации цен через два года на благоприятном уровне организация получит возможность перестроить производство под использование нового сырья, для чего в конце второго года потребуется инвестировать 100 млн руб. При этом переключение на использование нового сырья предполагается необратимым.

Таким образом, в случае принятия проекта $X$ в ситуации 3 организация в течение первых двух лет будет располагать реальным опционом, стоимость которого можно оценить аналогично европейскому опциону колл со сроком исполнения 2 года по формуле Магрейба [12; 20]. В качестве цены исполнения опциона возьмем сумму приведенных на начало 3-го года будущих маржинальных прибылей после налогообложения при использовании традиционного сырья и инвестиций на переключение производства на новое сырье. В качестве базового актива примем сумму приведенных на начало 3-го года будущих маржинальных прибылей при использовании нового сырья. По причине большей рискованности базового актива в первые два года для получения его текущей стоимости следует использовать более высокую ставку дисконтирования. Примем ее на уровне 20\%. Среднее квадратическое отклонение денежных потоков при использовании традиционного сырья составляет 0,15; при использовании нового сырья - 0,4; коэффициент корреляции цен на традиционное и новое сырье $-0,6$. 


\section{Результаты}

Результаты оценки ожидаемых денежных потоков от операционной деятельности по проектам $Y$ и $X$ в ситуации 1, полученные по формулам (1) и (8), представлены в табл. 3 и 4. Проведя дисконтирование денежных потоков проекта $Y$ и сложив их приведенные стоимости, получим стоимость проекта $Y$, равную 2098,4 млн руб. Стоимость проекта $X$ при условии его одновременной реализации с проектом $Y$ составит 1434,1 млн руб. Примечательно, при оценке изменения маржинальной прибыли по формулам (5-7), стоимость проекта $X$ будет существенно ниже и составит только 972,3 млн руб. Разница между двумя последними величинами составляет 461,8 млн руб. и является синергетическим эффектом. Он может быть получен при совместной реализации проектов, в первую очередь, за счет последовательного снятия двух ограничений по мощности на участках $X$ и $Y$.

В ситуациях 2 и 3 результаты анализа денежных потоков от операционной деятельности по проектам $Y$ и $X$ аналогичны результатам в ситуации 1 во всем, за исключением экономии на налоге на прибыль за счет изменения амортизации основных средств. NPV проектов $X$ и $Y$ в ситуациях 1-3, полученные на основе этих расчетов и данных о стартовых вложениях, приведены в табл. 5.

Как видно из величины по стр. 2 табл. 5, при рассмотрении проекта $X$ вне комплекса проектов он не имеет смысла во всех трех ситуациях, поскольку реализации его потенциала будет препятствовать ограничение по мощности на участке $Y$. На этом сходство между данными ситуациями заканчивается.

В ситуации 1 снятие ограничения по мощности участка $Y$ в сочетании с улучшением показателей эффективности производства позволяет увеличить рыночную добавленную стоимость бизнеса на 148,4 млн руб. Принятие же проекта $X$ (при условии его одновременной реализации с проектом $Y$ ) позволит увеличить этот показатель еще на 84,2 млн руб. Таким образом, в этой ситуации оценки проектов, получаемые в последовательности согласно ТОС, дают исчерпывающую информацию об их реальной ценности для организации.

Тем не менее, этого нельзя сказать о ситуации 2. В этой ситуации проект $Y$, выступающий с позиций ТОС в качестве условия принятия проекта $X$, имеет отрицательную NPV (-112,3 млн руб.). В то же время NPV проекта $X$ (при условии принятия проекта Y) положительна и составляет 344,9 млн руб. Таким образом, ограничивая себя анализом этих проектов в предписываемой ТОС последовательности, мы рискуем целиком отклонить выгодную инвестиционную стратегию еще на этапе рассмотрения проекта $Y$, если не будем принимать во внимание оценку проекта $X$ на втором этапе ее формирования.

В ситуации 3 проект Y имеет то же NPV, что и в ситуации 1, и может быть признан целесообразным вне зависимости от намерений руководства предприятия принять или отклонить проект $X$. Тем не менее, проект $X$, рассматриваемый вне контекста возможных будущих изменений системы, имеет отрицательное NPV (-89,6 млн руб.) и может быть отклонен. Однако если во внимание будет принята стоимость опциона на замену сырья при благоприятной рыночной конъюнктуре в будущем, оценка проекта $X$ должна быть иной. Поскольку стоимость этого опциона составляет 157,1 млн руб. и превышает прямые потери от проекта, его отклонение приведет к появлению упущенных выгод по крайней мере на 67,5 млн руб. Таким образом, прямолинейное следование ТОС в оценке проекта $X$ без учета его скрытых возможностей, также способно приводить к негативным последствиям. 


\section{Денежные потоки от операционной деятельности} по проекту $Y$ в ситуации 1 , млн руб.

Table 3. Cash flows from operating activity under the project $Y$ in case 1, millions rub.

\begin{tabular}{|l|c|c|c|}
\hline \multicolumn{1}{|c|}{ Наименование показателя } & Год 1 & Год 2 & $\begin{array}{c}\text { Начиная } \\
\text { с года 3 }\end{array}$ \\
\hline $\begin{array}{l}\text { Изменение маржинальной прибыли после налогообло- } \\
\text { жения, в том числе за счет: }\end{array}$ & 336,0 & 336,0 & 336,0 \\
\hline $\begin{array}{l}\text { снижения нормы затрат полуфабриката в связи с из- } \\
\text { менением технологии производста }\end{array}$ & 46,8 & 46,8 & 46,8 \\
\hline $\begin{array}{l}\text { увеличения производственной мощности за счет уве- } \\
\text { личения нормы выхода конечного продукта и рас- } \\
\text { ширения емкости технологического оборудования на } \\
\text { входе в процесс }\end{array}$ & 289,2 & 289,2 \\
\hline $\begin{array}{l}\text { Экономия на эксплуатационных условно-постоянных } \\
\text { расходах (после налогообложения) }\end{array}$ & 16,0 & 16,0 & 16,0 \\
\hline $\begin{array}{l}\text { Экономия на налоге на прибыль в связи с изменени- } \\
\text { ем амортизации основных средств }\end{array}$ & 48,8 & 48,8 & 48,8 \\
\hline $\begin{array}{l}\text { Итого денежный поток от операционной деятельности } \\
\text { по проекту }\end{array}$ & 400,8 & 400,8 & 400,8 \\
\hline
\end{tabular}

Источник: авторская разработка.

Таблица 4

Денежные потоки от операционной деятельности по проекту $X$ (при условии принятия проекта $Y$ ) в ситуации 1 , млн руб. Table 4. Cash flows from operating activity under project $X$ (subject to the adoption of project $Y$ ) in case 1, millions rub.

\begin{tabular}{|l|c|c|c|}
\hline \multicolumn{1}{|c|}{ Наименование показателя } & Год 1 & Год 2 & $\begin{array}{c}\text { Начиная } \\
\text { с года 3 }\end{array}$ \\
\hline $\begin{array}{l}\text { Изменение маржинальной прибыли после налого- } \\
\text { обложения, в том числе за счет: }\end{array}$ & 95,5 & 199,3 & 251,2 \\
\hline $\begin{array}{l}\text { снижения нормы затрат сырья на выработку полу- } \\
\text { фабриката }\end{array}$ & 68,0 & 68,0 & 68,0 \\
\hline $\begin{array}{l}\text { снижения нормы затрат полуфабриката в связи с улуч- } \\
\text { шением качества полуфабриката }\end{array}$ & 12,0 & 12,0 & 12,0 \\
\hline $\begin{array}{l}\text { увеличения производственной мощности за счет уве- } \\
\text { личения нормы выхода конечного продукта и рас- } \\
\text { ширения емкости технологического оборудования на } \\
\text { входе в процесс }\end{array}$ & 119,3 & 171,2 \\
\hline $\begin{array}{l}\text { Экономия на эксплуатационных условно-постоянных } \\
\text { расходах (после налогообложения) }\end{array}$ & 24,0 & 24,0 & 24,0 \\
\hline $\begin{array}{l}\text { Экономия на налоге на прибыль в связи с изменени- } \\
\text { ем амортизации основных средств }\end{array}$ & 33,8 & 33,8 & 33,8 \\
\hline $\begin{array}{l}\text { Итого денежный поток от операционной деятельности } \\
\text { по проекту }\end{array}$ & 153,3 & 257,0 & 308,9 \\
\hline
\end{tabular}

Источник: авторская разработка. 
Показатели изменения стоимости предприятия при запуске проектов $X$ и $Y$ в ситуациях 1-3, млн руб.

Table 5. Changes in business value due to the adoption of projects $\mathrm{X}$ and $\mathrm{Y}$ in cases $1-3$, million rub.

\begin{tabular}{|l|c|c|c|}
\hline \multicolumn{1}{|c|}{ Наименование показателя } & Ситуация 1 & Ситуация 2 & Ситуация 3 \\
\hline NPV проекта на участке $Y$ & 148,4 & $-112,3$ & 148,4 \\
\hline $\begin{array}{l}\text { NPV проекта на участке } X \text { при его изоли- } \\
\text { рованном рассмотрении вне комплекса про- } \\
\text { ектов }\end{array}$ & $-377,7$ & $-116,9$ & $-551,5$ \\
\hline $\begin{array}{l}\text { NPV проекта на участке } X \text { при условии его } \\
\text { совместной реализации с проектом Y без уче- } \\
\text { та реального опциона }\end{array}$ & 84,2 & 344,9 & $-89,6$ \\
\hline $\begin{array}{l}\text { Стоимость реального опциона, получаемого } \\
\text { при условии запуска проекта } X\end{array}$ & 0 & 0 & 157,1 \\
\hline $\begin{array}{l}\text { NРV проекта на участке } X \text { при условии его } \\
\text { совместной реализации с проектом Y, скор- } \\
\text { ректированная на стоимость опциона }\end{array}$ & 84,2 & 344,9 & 67,5 \\
\hline $\begin{array}{l}\text { Общее приращение стоимости предприятия } \\
\text { в результате реализации комплекса проектов }\end{array}$ & 232,6 & 232,6 & 215,9 \\
\hline
\end{tabular}

Источник: авторская разработка.

\section{Обсуждение и выводы}

По результатам проведенного исследования можно сделать следующие выводы.

Первое ограничение применимости ТОС в стратегическом анализе заключается в том, что в некоторых ситуациях увеличение рыночной добавленной стоимости бизнеса может создаваться только комбинацией нескольких мероприятий, в частности, инвестиционных проектов, приводящих к последовательному снятию ограничений и созданию значительных положительных синергетических эффектов (что видно из ситуации 2). В таких ситуациях каждое мероприятие в ряду стратегических преобразований не может быть отдельным объектом анализа и должно оцениваться в контексте не только предыдущих, но и последующих планируемых мероприятий. Поэтому в общем случае пять направляющих шагов ТОС оправданно использовать для определения последовательности преобразований, но их оценка в этой же последовательности на цепной основе может занизить их подлинную ценность и привести к полному или частичному необоснованному отклонению стратегии.

Второе ограничение заключается в том, что ценность мероприятия в ряду изменений хозяйственной системы, выбираемых в соответствии с пятью направляющими шагами ТОС, может определяться не только его прямыми последствиями, но и теми изменениями стратегии, которые компания получит возможность совершить при условии его выполнения (что видно из ситуации 3). Тем не менее, часто эти возможности скрыты и трудно поддаются оценке, несмотря на успехи теории опционов. Кроме того, эти возможности иногда сами порождают другие возможности (т.е. создают опцион на опцион), которые еще менее явны. Поэтому существует риск недооценки отдельных проектов или всего плана стратегических преобразований при их рассмотрении с позиций ТОС. 
В связи с этим объектом оценки в стратегическом анализе должен быть не каждый последующий цикл пяти направляющих шагов ТОС, а весь ряд будущих преобразований с учетом порождаемых ими реальных опционов, т.е. стратегия в целом, как это предусматривается традиционным стратегическим менеджментом. Это ослабит действие указанных ограничений, хотя, естественно, никогда не позволит снять их полностью в связи с постоянно присутствующей в бизнесе неопределенностью. Прямолинейное же применение пяти направляющих шагов ТОС в стратегическом анализе может привести к возникновению упущенных выгод в результате занижения подлинной ценности проектов и стратегий.

Тем не менее, использование ТОС в стратегическом анализе может помочь предотвратить принятие проектов, не приводящих к снятию в стратегической перспективе ни одного потенциального ограничения и разрушающих рыночную добавленную стоимость бизнеса. Поэтому применение подхода ТОС к работе с ограничениями следует рассматривать как необходимое, но не достаточное условие разработки успешной стратегии.

\section{Литература}

1. Балашова Е. С. Современные модели ресурсного менеджмента промышленного предприятия // Информационно-управляющие системы. 2015. Т. 74. № 1. С. 105-111.

2. Детмер У. Теория ограничений Голдратта: Системный подход к непрерывному совершенствованию. М. : Альпина Паблишер, 2017.

3. Детмер У., Шрагенхайм Э. Производство с невероятной скоростью: улучшение финансовых результатов предприятия. М. : Альпина Паблишер, 2009.

4. Ковалев В.В. Финансовый менеджмент: теория и практика. М. : Проспект, 2017.

5. Корбетт Т. Управленческий учет по ТОС. Киев : НіД, 2009.

6. Лич Л. Вовремя и в рамках бюджета: Управление проектами по методу критической цепи. М. : Альпина Паблишер, 2017.

7. Хайруллина М.В., Кислицына О.А., Чуваев А.В. Непрерывное улучшение производственной системы промышленного предприятия: показатели и модель оценки // Научно-технические ведомости Санкт-Петербургского государственного политехнического университета. Экономические науки. 2015. Т. 233. № 6. С. 81-90.

8. Шрагенхайм Э. Управленческие дилеммы. Теория ограничений в действии. М. : Альпина Бизнес Букс, 2007.

9. Bragg S. M. Throughput Accounting. A Guide to Constraint Management. Hoboken : John Wiley and Sons, 2007.

10. Chaudhari C.V., Mukhopadhyay S.K. Application of Theory of Constraints in an integrated poultry industry // International journal of production research. 2003. Vol. 41. N 4. P. 799-817.

11. Dias R. M.F., Silva L.D., Tenera A. Application of a Proposed TLS Model in a Lean Productive System // Independent Journal of Management and Production. 2019. Vol. 10. № 1. P. 76-100.

12. Fischer S. Call Option Pricing When the Exercise Price is Uncertain, and the Valuation of Index Bonds // Journal of Finance. 1978. Vol. 33. N 1. P. 169-176.

13. Goldratt E. It's Not Luck. Great Barrington, The North River Press, 1994.

14. Goldratt E. The Haystack Syndrome: Sifting Information Out of the Data Ocean. Croton-onHudson : The North River Press, 1990.

15. Goldratt E., Cox J. The Goal. A Process of Ongoing Improvement. Great Barrington : The North River Press, 2004.

16. Goldratt E., Schragenheim E., Ptak C. Necessary but not Sufficient. Great Barrington : The North River Press, 2000.

17. Gundogar E., Sari M., Kokcam A. H. Dynamic bottleneck elimination in mattress manufacturing line using theory of constraints // Springerplus. 2016. Vol. 5 [Электронный ресурс]. URL: https://doi.org/10.1186/s40064-016-2947-1 (дата обращения: 24.07.2019).

18. Gupta M., Boyd L. An Excel-based dice game: an integrative learning activity in operations management // International Journal of Operations and Production Management. 2011. Vol. 31. N 6. P. 608-630.

19. Gupta M. C., Sahi G. K., Chahal H. Improving market orientation: the theory of constraints-based framework // Journal of Strategic Marketing. 2013. Vol. 21. N 4. P. 305-322. 
20. Margrabe W. The Value of an Option to Exchange One Asset for Another // Journal of Finance. 1978. Vol. 33. N 1. P. 177-186.

21. Mokgatlha S., Kadama F.K. Constraints in the pharmaceutical supply chain to clinics in the Mafikeng area, South Africa // WIT Transactions on Ecology and the Environment. 2018. Vol. 226. P. 449-457.

22. Perez-Campdesuner R., Perez-Pravia M., Sanchez-Rodriguez A. et al. Application of a Methodology Based on the Theory of Constraints in the Sector of Tourism Services // Journal of Industrial Engineering and Management. 2017. Vol. 10. N 1. P. 7-27.

23. Petrochenko M. V., Velichkin V.Z., Kazakov Y. N. et al. Reliability assessment of the construction schedule by the critical chain method // Magazine of Civil Engineering. 2018. Vol. 81. N 5. P. 25-31.

24. Pretorius $P$. Introducing in-between decision points to TOC's five focusing steps // International Journal of Production Research. 2014. Vol. 52. N 2. P. 496-506.

25. Puche J., Ponte B., Costas J. et al. Systemic approach to supply chain management through the viable system model and the theory of constraints // Production Planning and Control. 2016. Vol. 27. N 5. P. 421-430.

26. Sproull B. Maximizing Profits through the Integration of Lean, Six Sigma and the Theory of Constraints. New York : Productivity Press, 2009.

\section{Об авторе:}

Нечитайло Игорь Алексеевич, доцент Высшей инженерно-экономической школы СанктПетербургского политехнического университета Петра Великого (Санкт-Петербург, Российская Федерация), кандидат экономических наук, доцент; nechitajlo_ia@spbstu.ru

\section{References}

1. Balashova E.S. Modern models of resource management of an industrial enterprise // Information and Control Systems [Informacionno-upravljajushhie sistemy]. 2015. Vol. 74. N 1. P. 105-111. (In rus.)

2. Dettmer W. Goldratt's Theory of Constraints. A Systems Approach to Continuous Improvement. M. : Alpina Publisher, 2017. (In rus.)

3. Dettmer W., Schragenheim E. Manufacturing at Warp Speed. Optimizing Supply Chain Financial Performance. M. : Alpina Publisher, 2009. (In rus.)

4. Kovalev V. V. Financial management: theory and practice. M., Prospect Publisher, 2017. (In rus.)

5. Corbett T. Throughput Accounting: TOC's Management Accounting System. Kiev : NiD, 2009. (In rus.)

6. Leach L. Critical Chain Project Management. M. : Alpina Publisher, 2017. (In rus.)

7. Khairullina M. V., Kislitsyna O.A., Chuvaev A. V. Continuous improvement of the industrial enterprise production system: indicators and evaluation model. SPbPU Journal. Economics [Nauchno-tehnicheskie vedomosti Sankt-Peterburgskogo gosudarstvennogo politehnicheskogo universiteta Jekonomicheskie nauki]. 2015. Vol. 233. N 6. P. 81-90. (In rus.)

8. Schragenheim E. Management Dilemmas. The Theory of Constraints Approach to Problem Identification and Solutions. M. : Alpina Business Books, 2007. (In rus.)

9. Bragg S. M. Throughput Accounting. A Guide to Constraint Management. Hoboken : John Wiley and Sons, 2007.

10. Chaudhari C.V., Mukhopadhyay S.K. Application of Theory of Constraints in an integrated poultry industry // International journal of production research. 2003. Vol. 41. N 4. P. 799-817.

11. Dias R. M.F., Silva L.D., Tenera A. Application of a Proposed TLS Model in a Lean Productive System // Independent Journal of Management and Production. 2019. Vol. 10. N 1. P. 76-100.

12. Fischer S. Call Option Pricing When the Exercise Price is Uncertain, and the Valuation of Index Bonds // Journal of Finance. 1978. Vol. 33. N 1. P. 169-176.

13. Goldratt E. It's Not Luck. Great Barrington, The North River Press, 1994.

14. Goldratt E. The Haystack Syndrome: Sifting Information Out of the Data Ocean. Croton-onHudson : The North River Press, 1990.

15. Goldratt E., Cox J. The Goal. A Process of Ongoing Improvement. Great Barrington : The North River Press, 2004.

16. Goldratt E., Schragenheim E., Ptak C. Necessary but not Sufficient. Great Barrington : The North River Press, 2000. 
17. Gundogar E., Sari M., Kokcam A. H. Dynamic bottleneck elimination in mattress manufacturing line using theory of constraints // Springerplus. 2016. Vol. 5 [Elecronic resours]. URL: https:// doi.org/10.1186/s40064-016-2947-1 (accessed: 24.07.2019)

18. Gupta M., Boyd L. An Excel-based dice game: an integrative learning activity in operations management // International Journal of Operations and Production Management. 2011. Vol. 31. N 6. P. 608-630.

19. Gupta M.C., Sahi G. K., Chahal H. Improving market orientation: the theory of constraints-based framework // Journal of Strategic Marketing. 2013. Vol. 21. N 4. P. 305-322.

20. Margrabe W. The Value of an Option to Exchange One Asset for Another // Journal of Finance. 1978. Vol. 33. N 1. P. 177-186.

21. Mokgatlha S., Kadama F.K. Constraints in the pharmaceutical supply chain to clinics in the Mafikeng area, South Africa // WIT Transactions on Ecology and the Environment. 2018. Vol. 226. P. 449-457.

22. Perez-Campdesuner R., Perez-Pravia M., Sanchez-Rodriguez A. et al. Application of a Methodology Based on the Theory of Constraints in the Sector of Tourism Services // Journal of Industrial Engineering and Management. 2017. Vol. 10. N 1. P. 7-27.

23. Petrochenko M. V., Velichkin V.Z., Kazakov Y.N. et al. Reliability assessment of the construction schedule by the critical chain method // Magazine of Civil Engineering. 2018. Vol. 81. N 5. P. 25-31.

24. Pretorius P. Introducing in-between decision points to TOC's five focusing steps // International Journal of Production Research. 2014. Vol. 52. N 2. P. 496-506.

25. Puche J., Ponte B., Costas J. et al. Systemic approach to supply chain management through the viable system model and the theory of constraints // Production Planning and Control. 2016. Vol. 27. N 5. P. 421-430.

26. Sproull B. Maximizing Profits through the Integration of Lean, Six Sigma and the Theory of Constraints. New York : Productivity Press, 2009.

\section{About the author:}

Igor A. Nechitaylo, Associate professor of Peter the Great St. Petersburg Polytechnic University (Saint-Petersburg, Russian Federation), PhD in Economics, Associate professor; nechitajlo_ia@ spbstu.ru 